\title{
Extremely non-normal continued fractions
}

\author{
by \\ L. Olsen (St. Andrews)
}

1. Introduction and statement of results. Let $\mathbb{P}$ denote the irrational numbers in the closed unit interval, i.e.

$$
\mathbb{P}:=[0,1] \backslash \mathbb{Q} \text {. }
$$

For $x \in \mathbb{P}$, let

$$
x=\frac{1}{a_{1}(x)+\frac{1}{a_{2}(x)+\frac{1}{a_{3}(x)+\ldots}}}
$$

where $a_{n}(x) \in \mathbb{N}$ for all $n$, denote the simple (infinite) continued fraction expansion of $x$. For a positive integer $n$ and a digit $i \in \mathbb{N}$, we write

$$
\Pi(x, i ; n)=\frac{\left|\left\{1 \leq j \leq n \mid a_{j}(x)=i\right\}\right|}{n}
$$

for the frequency of the digit $i$ among the first $n$ digits in the continued fraction expansion of $x$. A classical result due to Lévy [Lé] says that for Lebesgue almost all $x \in \mathbb{P}$ we have

$$
\Pi(x, i ; n) \rightarrow \frac{1}{\log 2} \log \frac{(i+1)^{2}}{i(i+2)}
$$

for all $i \in \mathbb{N}$; the reader is referred to the textbook [Bi, p. 45] for a contemporary proof of this based on the ergodic theorem. In analogy with normal numbers (cf. $[\mathrm{KN}]$ ), we will say that a number $x \in \mathbb{P}$ is continued fraction normal (c-f-normal) if it satisfies (1.2). Hence, using this terminology, Lévy's result says that Lebesgue almost all $x \in \mathbb{P}$ are c-f-normal.

2000 Mathematics Subject Classification: Primary 11K50; Secondary 11K16, 30B70, $28 \mathrm{~A} 80$.

Key words and phrases: continued fractions, divergence points, Baire category, Hausdorff dimension, packing dimension. 
In this paper we will prove that from a topological viewpoint, most numbers fail to be c-f-normal in a very spectacular way. We will show that (in the Baire sense) most numbers are as far away from being c-f-normal as possible. Similar results for sets of numbers whose $N$-adic expansion/Lüroth expansion deviates significantly from the $N$-adic expansion/Lüroth expansion of Lebesgue almost all numbers have been obtained by Olsen [Ol2] and Šalát [Ša1].

We first introduce some notation. For a positive integer $n$ and a finite string $\mathbf{i}=i_{1} \ldots i_{k} \in \mathbb{N}^{k}$ of length $k$ with entries $i_{j} \in \mathbb{N}$, we write

$$
\Pi(x, \mathbf{i} ; n)=\frac{\left|\left\{1 \leq j \leq n \mid a_{j}(x)=i_{1}, \ldots, a_{j+k-1}(x)=i_{k}\right\}\right|}{n}
$$

for the frequency of the string $\mathbf{i}$ among the first $n$ digits in the simple continued fraction expansion of $x$, and let

$$
\Pi_{k}(x ; n)=(\Pi(x, \mathbf{i} ; n))_{\mathbf{i} \in \mathbb{N}^{k}}
$$

denote the vector of frequencies $\Pi(x, \mathbf{i} ; n)$ of all strings $\mathbf{i} \in \mathbb{N}^{k}$ of length $k$. We define the subset $\Delta_{k}$ of $\ell^{1}$ by

$$
\Delta_{k}=\left\{\left(p_{\mathbf{i}}\right)_{\mathbf{i} \in \mathbb{N}^{k}} \mid p_{\mathbf{i}} \geq 0, \sum_{\mathbf{i}} p_{\mathbf{i}}=1\right\},
$$

i.e. $\Delta_{k}$ denotes the simplex of probability vectors indexed by strings $\mathbf{i}=$ $i_{1} \ldots i_{k}$ of length $k$ with entries $i_{j} \in \mathbb{N}$. We will always equip $\Delta_{k}$ with the 1-norm $\|\cdot\|_{1}$. The vector $\Pi_{k}(x ; n)$ of frequencies of strings of length $k$ among the first $n$ digits in the simple continued fraction expansion of $x$ clearly belongs to $\Delta_{k}$. We will quantify the non-normality of $x$ by considering the extent to which the sequence $\left(\Pi_{k}(x ; n)\right)_{n}$ fills up the simplex $\Delta_{k}$. Of course, in general, it is not true that the sequence $\left(\Pi_{k}(x ; n)\right)_{n}$ fills up a substantial part of $\Delta_{k}$ for any $x$. For example, consider strings of length 3. By considering all possible ways a string of length 2 , such as $37 \in \mathbb{N}^{2}$ (i.e. 37 represents the string of length 2 whose first digit equals 3 and whose second digit equals 7 ), can arise it is easily seen that

$$
\left|\sum_{i \in \mathbb{N}} \Pi(x, i 37 ; n)-\sum_{i \in \mathbb{N}} \Pi(x, 37 i ; n)\right| \leq \frac{1}{n}
$$

for all $x$. This implies that for each $x$, all but finitely many points in the sequence $\left(\Pi_{3}(x ; n)\right)_{n}$ will be very close to the subsimplex

$$
\Delta_{3} \cap\left\{\left(x_{\mathbf{i}}\right)_{\mathbf{i} \in \mathbb{N}^{3}} \in \ell^{1} \mid \sum_{i \in \mathbb{N}} x_{i 37}=\sum_{i \in \mathbb{N}} x_{37 i}\right\} .
$$

Hence, in general the sequence $\left(\Pi_{k}(x ; n)\right)_{n}$ will not fill up a significant part of the simplex $\Delta_{k}$, and the full simplex $\Delta_{k}$ is not the "correct" object to consider. Rather we need to consider the subsimplex defined by slicing $\Delta_{k}$ 
by various planes corresponding to the subsimplex in (1.3). Motivated by this, we define the subsimplex $S_{k}$ of shift invariant probability vectors in $\mathbb{R}^{\mathbb{N}^{k}}$ by

$$
\begin{aligned}
\mathrm{S}_{k}=\left\{\left(p_{\mathbf{i}}\right)_{\mathbf{i} \in \mathbb{N}^{k}} \mid p_{\mathbf{i}} \geq 0, \sum_{\mathbf{i}} p_{\mathbf{i}}=1, \sum_{i} p_{i \mathbf{i}}=\sum_{i} p_{\mathbf{i} i}\right. & \text { for all } \left.\mathbf{i} \in \mathbb{N}^{k-1}\right\} .
\end{aligned}
$$

Observe that $\Delta_{1}=\mathrm{S}_{1}$. We will now prove that the subsimplex $\mathrm{S}_{k}$ is the "correct" object. Let $\mathrm{A}_{k}(x)$ denote the set of accumulation points of the sequence $\left(\Pi_{k}(x ; n)\right)_{n}$ with respect to $\|\cdot\|_{1}$, i.e.

$$
\begin{array}{r}
\mathrm{A}_{k}(x)=\left\{\mathbf{p} \in \Delta_{k} \mid \text { there exists a subsequence }\left(\Pi_{k}\left(x ; n_{m}\right)\right)_{m}\right. \\
\text { such that } \left.\left\|\Pi_{k}\left(x ; n_{m}\right)-\mathbf{p}\right\|_{1} \rightarrow 0\right\} .
\end{array}
$$

The next result says that the subsimplex $S_{k}$ is, indeed, the "correct" simplex to consider: all accumulation points of $\left(\Pi_{k}(x ; n)\right)_{n}$ belong to $S_{k}$.

Theorem 0. Let $x \in[0,1]$. Then

$$
\mathrm{A}_{k}(x) \subseteq \mathrm{S}_{k}
$$

Proof. Let $\mathbf{p}=\left(p_{\mathbf{i}}\right)_{\mathbf{i} \in \mathbb{N}^{k}}$ be an accumulation point of the sequence $\left(\Pi_{k}(x ; n)\right)_{n}$ with respect to $\|\cdot\|_{1}$. We can thus find a strictly increasing sequence $\left(n_{m}\right)_{m}$ of positive integers such that

$$
\left\|\Pi_{k}\left(x ; n_{m}\right)-\mathbf{p}\right\|_{1} \rightarrow 0 .
$$

By considering all possible ways a string $\mathbf{i} \in \mathbb{N}^{k-1}$ of length $k-1$ can arise it follows that

$$
\left|\sum_{i \in \mathbb{N}} \Pi(x, i \mathbf{i} ; n)-\sum_{i \in \mathbb{N}} \Pi(x, \mathbf{i} i ; n)\right| \leq 1 / n .
$$

It follows from (1.6) and (1.7) that if $\mathbf{i} \in \mathbb{N}^{k-1}$, then

$$
\begin{aligned}
\left|\sum_{i \in \mathbb{N}} p_{i \mathbf{i}}-\sum_{i \in \mathbb{N}} p_{\mathbf{i} i}\right| \leq & \left|\sum_{i \in \mathbb{N}} p_{i \mathbf{i}}-\sum_{i \in \mathbb{N}} \Pi\left(x, i \mathbf{i} ; n_{m}\right)\right| \\
& +\left|\sum_{i \in \mathbb{N}} \Pi\left(x, i \mathbf{i} ; n_{m}\right)-\sum_{i \in \mathbb{N}} \Pi\left(x, \mathbf{i} i ; n_{m}\right)\right| \\
& +\left|\sum_{i \in \mathbb{N}} \Pi\left(x, \mathbf{i} i ; n_{m}\right)-\sum_{i \in \mathbb{N}} p_{\mathbf{i} i}\right| \\
\leq & \left\|\Pi_{k}\left(x ; n_{m}\right)-\mathbf{p}\right\|_{1}+1 / n_{m}+\left\|\Pi_{k}\left(x ; n_{m}\right)-\mathbf{p}\right\|_{1} \rightarrow 0 .
\end{aligned}
$$

This implies that $\sum_{i \in \mathbb{N}} p_{i \mathbf{i}}=\sum_{i \in \mathbb{N}} p_{\mathbf{i} i}$ for all $\mathbf{i} \in \mathbb{N}^{k-1}$.

We will say that the number $x$ is extremely non-k-continued fraction normal (extremely non- $k$-c-f-normal) if the set of accumulation points of 
the sequence $\left(\Pi_{k}(x ; n)\right)_{n}$ (with respect to $\left.\|\cdot\|_{1}\right)$ equals $\mathrm{S}_{k}$, and we will denote the set of extremely non- $k$-c-f-normal numbers by $\mathbb{E}_{k}$, i.e.

$$
\mathbb{E}_{k}=\left\{x \in \mathbb{P} \mid \mathrm{A}_{k}(x)=\mathrm{S}_{k}\right\} .
$$

We will say that a number is extremely non-continued fraction normal (extremely non-c-f-normal) if it is extremely non- $k$-c-f-normal for all $k$. We let $\mathbb{E}$ denote the set of extremely non-c-f-normal numbers, i.e.

$$
\mathbb{E}=\bigcap_{k} \mathbb{E}_{k}
$$

Hence, the numbers in $\mathbb{E}$ are as far away from being c-f-normal as possible. Our main result (Theorem 1 below) states, somewhat surprisingly, that the set $\mathbb{E}$ is extremely big from a topological viewpoint.

Theorem 1. (1) The set $\mathbb{E}$ is comeager in $\mathbb{P}$, i.e. $\mathbb{P} \backslash \mathbb{E}$ is of the first category $($ in $\mathbb{P})$. In particular, $\mathbb{E}$ is of the second category $($ in $\mathbb{P})$.

(2) The set $\mathbb{E}$ is comeager in $[0,1]$, i.e. $[0,1] \backslash \mathbb{E}$ is of the first category $($ in $[0,1])$. In particular, $\mathbb{E}$ is of the second category $($ in $[0,1])$.

Theorem 1 shows that from a topological point of view, a typical number in $[0,1]$ is as far away from being c-f-normal as possible. The proof of Theorem 1 is given in Section 2.

Define the set $\mathbb{S}$ by

$\mathbb{S}=\left\{x \in \mathbb{P} \mid\right.$ the sequence $(\Pi(x, i ; n))_{n}$ is dense in $[0,1]$ for all $\left.i \in \mathbb{N}\right\}$.

Šalát $[\breve{S} a 2]$ proved that $\mathbb{S}$ is comeager. Since clearly $\mathbb{E} \subseteq \mathbb{E}_{1} \subseteq \mathbb{S}$ it follows immediately from Theorem 1 that $\mathbb{S}$ is comeager.

As an immediate corollary to Theorem 1, we obtain the packing dimension $\operatorname{Dim} \mathbb{E}$ of $\mathbb{E}$; the reader is referred to $[\mathrm{Fa}]$ for the definition of Dim.

Corollary 2. Dim $\mathbb{E}=1$.

Proof. It follows from [Ed, Exercise (1.8.4)] that if $E$ is a subset of $\mathbb{R}$ with $\operatorname{Dim} E<1$, then $E$ is of the first category. This and Theorem 1 imply that $\operatorname{Dim} \mathbb{E}=1$.

Let dim denote the Hausdorff dimension; the reader is referred to [Fa] for the definition. We have not considered the problem of computing the Hausdorff dimension of the set $\mathbb{E}$. However, it follows from [Ol1, Ol2] that the Hausdorff dimension of the set of numbers whose $N$-adic expansion satisfies a similar condition of extreme non-normality equals 0 , and we therefore make the following conjecture.

ConjeCture $3 . \operatorname{dim} \mathbb{E}=0$. In fact, for each positive integer $k$, we have $\operatorname{dim} \mathbb{E}_{k}=0$. 
2. Proof of Theorem 1. We start by introducing some notation. Let $\mathbb{N}^{*}=\bigcup_{n} \mathbb{N}^{n}$, and define $\pi: \mathbb{N}^{\mathbb{N}} \rightarrow \mathbb{P}$ by

$$
\pi(\omega)=\frac{1}{\omega_{1}+\frac{1}{\omega_{2}+\frac{1}{\omega_{3}+\ldots}}}
$$

for $\omega=\omega_{1} \omega_{2} \ldots \in \mathbb{N}^{\mathbb{N}}$. If $\omega=\omega_{1} \omega_{2} \ldots \in \mathbb{N}^{\mathbb{N}}$ and $m$ is a positive integer or if $\omega=\omega_{1} \ldots \omega_{n} \in \mathbb{N}^{n}$ and $m$ is a positive integer with $m \leq n$, then we will write $\omega \mid m=\omega_{1} \ldots \omega_{m}$. For $\omega \in \mathbb{N}^{n}$, we write $|\omega|=n$ and we define the cylinder $[\omega]$ generated by $\omega$ by

$$
[\omega]=\left\{\sigma \in \mathbb{N}^{\mathbb{N}}|\sigma| n=\omega\right\} .
$$

For $\mathbf{i}=i_{1} \ldots i_{k} \in \mathbb{N}^{k}$ and $\omega=\omega_{1} \ldots \omega_{n+k-1} \in \mathbb{N}^{n+k-1}$ write

$$
\mathrm{P}(\omega, \mathbf{i})=\frac{\left|\left\{1 \leq i \leq n \mid \omega_{i}=i_{1}, \ldots, \omega_{i+k-1}=i_{k}\right\}\right|}{n}
$$

for the frequency of the string $\mathbf{i}$ among the digits of the string $\omega$. Let

$$
\mathrm{P}_{k}(\omega)=(\mathrm{P}(\omega, \mathbf{i}))_{\mathbf{i} \in \mathbb{N}^{k}}
$$

denote the vector of all frequencies of strings $\mathbf{i}$ of length $k$ among the digits of $\omega$.

We now turn towards the proof of Theorem 1. Let

$$
\begin{array}{r}
\mathrm{S}_{k}^{*}=\bigcup_{N}\left\{\left(p_{\mathbf{i}}\right)_{\mathbf{i} \in \mathbb{N}^{k}} \mid p_{\mathbf{i}} \geq 0, \sum_{\mathbf{i}} p_{\mathbf{i}}=1, \sum_{i} p_{i \mathbf{i}}=\sum_{i} p_{\mathbf{i} i} \text { for all } \mathbf{i} \in \mathbb{N}^{k-1},\right. \\
\left.p_{\mathbf{i}}=0 \text { for } \mathbf{i} \in \mathbb{N}^{k} \backslash\{1, \ldots, N\}^{k}\right\} .
\end{array}
$$

The set $S_{k}^{*}$ is clearly a dense and separable subset of $\left(S_{k},\|\cdot\|_{1}\right)$. We can therefore find a sequence $\left(\mathbf{q}_{k, m}\right)_{m}$ in $\mathbf{S}_{k}^{*}$ that is dense in $\left(\mathrm{S}_{k},\|\cdot\|_{1}\right)$. For positive integers $k$ and $m$ write

$$
\mathbb{E}_{k, m}=\left\{x \in \mathbb{P} \mid \mathbf{q}_{k, m} \text { is an accumulation point of }\left(\Pi_{k}(x ; n)\right)_{n}\right\} .
$$

We clearly have

$$
\mathbb{E}=\bigcap_{k, m} \mathbb{E}_{k, m},
$$

and it therefore suffices to prove that $\mathbb{E}_{k, m}$ is comeager for all $k$ and $m$. Therefore, fix positive integers $k$ and $m$. Since the set $\mathbb{P}$ of irrationals is a Baire space, in order to prove that $\mathbb{E}_{k, m}$ is a comeager subset of $\mathbb{P}$, it suffices to construct a set $E \subseteq \mathbb{P}$ satisfying the following three conditions:

(1) $E \subseteq \mathbb{E}_{k, m}$;

(2) $E$ is dense in $\mathbb{P}$;

(3) $E$ is a $\mathcal{G}_{\delta}$ set. 
We will now proceed to construct a set $E$ with the desired properties. Write

$$
\mathbf{q}_{k, m}=\mathbf{q}=\left(q_{\mathbf{i}}\right)_{\mathbf{i} \in \mathbb{N}^{k}} .
$$

Since $\mathbf{q}$ belongs to $\mathrm{S}_{k}^{*}$, there exists a positive integer $N$ such that $q_{\mathbf{i}}=0$ for $\mathbf{i} \in \mathbb{N}^{k} \backslash\{1, \ldots, N\}^{k}$. Put

$$
\begin{aligned}
& Z_{n}=\left\{\omega \in \bigcup_{l \geq k n N^{k}}\{1, \ldots, N\}^{l} \mid\left\|\mathrm{P}_{k}(\omega)-\mathbf{q}\right\|_{1} \leq \frac{1}{n}\right\}, \\
& \widehat{Z}_{n}=\left\{\omega \in \bigcup_{l \geq k n N^{k}}\{1, \ldots, N\}^{l} \mid\left\|\mathrm{P}_{k}(\omega)-\mathbf{q}\right\|_{1} \leq \frac{5}{n}\right\} .
\end{aligned}
$$

Furthermore, for subsets $W, W_{1}, \ldots, W_{n}$ of $\mathbb{N}^{*}$ and $\omega \in \mathbb{N}^{*}$ we will write

$$
\begin{gathered}
W_{1} \ldots W_{n}=\left\{\omega_{1} \ldots \omega_{n} \mid \omega_{i} \in W_{i}\right\}, \\
\omega W=\{\omega \sigma \mid \sigma \in W\}, \quad[W]=\{[\sigma] \mid \sigma \in W\} .
\end{gathered}
$$

Lemma 2.1. Let $n$ be a positive integer and $\omega \in \mathbb{N}^{*}$. Then there exists an integer $Q \geq n$ such that

$$
\omega \underbrace{Z_{n} \ldots Z_{n}}_{Q \text { times }} \subseteq \widehat{Z}_{n} .
$$

Proof. Let

$$
\sigma=\omega \sigma_{1} \ldots \sigma_{Q} \in \omega \underbrace{Z_{n} \ldots Z_{n}}_{Q \text { times }} \quad \text { with } \sigma_{i} \in Z_{n} .
$$

Write $\omega=\omega_{1} \ldots \omega_{s}$ and $M=\max _{i} \omega_{i}$. For each $\mathbf{i} \in \mathbb{N}^{k}$ we clearly have

$$
\frac{\sum_{i}\left|\sigma_{i}\right| \mathrm{P}\left(\sigma_{i}, \mathbf{i}\right)}{|\sigma|} \leq \mathbf{P}(\sigma, \mathbf{i}) \leq \frac{|\omega|+\sum_{i}\left|\sigma_{i}\right| \mathrm{P}\left(\sigma_{i}, \mathbf{i}\right)+Q k}{|\sigma|} .
$$

Since no $\mathbf{i} \in \mathbb{N}^{k} \backslash\{1, \ldots, N\}^{k}$ is a substring of any $\sigma_{i}$ (because $\sigma_{i} \in Z_{n}$ ), this implies that

$$
\begin{aligned}
\left\|\mathbf{P}_{k}(\sigma)-\mathbf{q}\right\|_{1} \leq & \left\|\mathrm{P}_{k}(\sigma)-\sum_{i} \frac{\left|\sigma_{i}\right|}{|\sigma|} \mathrm{P}_{k}\left(\sigma_{i}\right)\right\|_{1}+\left\|\sum_{i} \frac{\left|\sigma_{i}\right|}{|\sigma|} \mathrm{P}_{k}\left(\sigma_{i}\right)-\mathbf{q}\right\|_{1} \\
= & \sum_{\mathbf{i} \in\{1, \ldots, N\}^{k}}\left|\mathrm{P}(\sigma, \mathbf{i})-\sum_{i} \frac{\left|\sigma_{i}\right|}{|\sigma|} \mathrm{P}_{k}\left(\sigma_{i}\right)\right| \\
& +\sum_{\mathbf{i} \in \mathbb{N}^{k} \backslash\{1, \ldots, N\}^{k}}\left|\mathrm{P}(\sigma, \mathbf{i})-\sum_{i} \frac{\left|\sigma_{i}\right|}{|\sigma|} \mathrm{P}_{k}\left(\sigma_{i}\right)\right| \\
& +\left\|\sum_{i} \frac{\left|\sigma_{i}\right|}{|\sigma|} \mathrm{P}_{k}\left(\sigma_{i}\right)-\mathbf{q}\right\|_{1}
\end{aligned}
$$




$$
\begin{aligned}
\leq & \sum_{\mathbf{i} \in\{1, \ldots, N\}^{k}} \frac{|\omega|+Q k}{|\sigma|}+\sum_{\substack{\mathbf{i} \in \mathbb{N}^{k} \backslash\{1, \ldots, N\}^{k} \\
\mathrm{P}(\omega, \mathbf{i}) \neq 0}} \frac{|\omega|}{|\sigma|} \\
& +\left\|\sum_{i} \frac{\left|\sigma_{i}\right|}{|\sigma|} \mathrm{P}_{k}\left(\sigma_{i}\right)-\mathbf{q}\right\|_{1} \\
\leq & N^{k} \frac{|\omega|+Q k}{Q n k N^{k}}+M^{k} \frac{|\omega|}{Q n k N^{k}}+\left\|\sum_{i} \frac{\left|\sigma_{i}\right|}{|\sigma|} \mathrm{P}_{k}\left(\sigma_{i}\right)-\mathbf{q}\right\|_{1} \\
= & \frac{1}{n}+c \frac{1}{Q}+\left\|\sum_{i} \frac{\left|\sigma_{i}\right|}{|\sigma|} \mathrm{P}_{k}\left(\sigma_{i}\right)-\mathbf{q}\right\|_{1}
\end{aligned}
$$

where we have used the fact that $|\sigma| \geq \sum_{i=1}^{Q}\left|\sigma_{i}\right| \geq Q n k N^{k}$ and written

$$
c=\frac{|\omega|}{n k}\left(1+\frac{M^{k}}{N^{k}}\right) \text {. }
$$

Also

$$
\begin{aligned}
\left\|\sum_{i} \frac{\left|\sigma_{i}\right|}{|\sigma|} \mathrm{P}_{k}\left(\sigma_{i}\right)-\mathbf{q}_{k, m}\right\|_{1} \leq & \left\|\sum_{i} \frac{\left|\sigma_{i}\right|}{|\sigma|} \mathrm{P}_{k}\left(\sigma_{i}\right)-\sum_{i} \frac{\left|\sigma_{i}\right|}{\sum_{j}\left|\sigma_{j}\right|} \mathrm{P}_{k}\left(\sigma_{i}\right)\right\|_{1} \\
& +\left\|\sum_{i} \frac{\left|\sigma_{i}\right|}{\sum_{j}\left|\sigma_{j}\right|} \mathrm{P}_{k}\left(\sigma_{i}\right)-\mathbf{q}_{k, m}\right\|_{1} \\
\leq & \sum_{i}\left|\sigma_{i}\right|\left|\frac{1}{|\sigma|}-\frac{1}{\sum_{j}\left|\sigma_{j}\right|}\right| \\
& +\sum_{i} \frac{\left|\sigma_{i}\right|}{\sum_{j}\left|\sigma_{j}\right|}\left\|\mathrm{P}_{k}\left(\sigma_{i}\right)-\mathbf{q}_{k, m}\right\|_{1} \\
\leq & \sum_{i} \frac{\left|\sigma_{i}\right|}{\sum_{j}\left|\sigma_{j}\right|} \frac{|\omega|}{|\sigma|}+\sum_{i} \frac{\left|\sigma_{i}\right|}{\sum_{j}\left|\sigma_{j}\right|} \frac{1}{n} \\
= & \frac{|\omega|}{|\sigma|}+\frac{1}{n} \leq \frac{|\omega|}{Q n k N^{k}}+\frac{1}{n} .
\end{aligned}
$$

It follows from (2.1) and (2.2) that

$$
\left\|\mathrm{P}_{k}(\omega)-\mathbf{q}\right\|_{1} \leq \frac{1}{n}+c \frac{1}{Q}+\frac{|\omega|}{Q n k N^{k}}+\frac{1}{n} .
$$

Hence, by choosing $Q$ large enough we can ensure that $Q \geq n$ and $\left\|\mathrm{P}_{k}(\omega)-\mathbf{q}\right\|_{1} \leq 5 / n$.

LEMma 2.2. There exist functions $u_{n}: \mathbb{N}^{*} \rightarrow \mathbb{N}^{*}, Q_{n}: \mathbb{N}^{*} \rightarrow \mathbb{N}$, with the following properties: for all $\omega \in \mathbb{N}^{*}$ we have

$$
\pi\left(\left[u_{n}(\omega)\right]\right)^{-} \subseteq \pi([\omega])^{\circ},
$$




$$
\begin{gathered}
u_{n}(\omega) \underbrace{Z_{n} \ldots Z_{n}}_{Q_{n}(\omega) \text { times }} \subseteq \widehat{Z}_{n}, \\
Q_{n}(\omega) \geq n .
\end{gathered}
$$

In (2.3), the closure and interior are with respect to the space $\mathbb{P}$.

Proof. Let $\omega \in \mathbb{N}^{*}$. Now choose $\sigma \in \pi^{-1}(\pi([\omega 22]))$. Hence $\pi(\sigma) \in$ $\pi([\omega 22])$, and we can thus choose a positive integer $m$ such that $\pi([\sigma \mid m]) \subseteq$ $\pi([\omega 2])^{-} \subseteq \pi([\omega])^{\circ}$. We now define $u_{n}(\omega)$ by $u_{n}(\omega)=\sigma \mid m$. Also, by Lemma 2.1 we can find an integer $Q_{n}(\omega)$ such that (2.4) and (2.5) are satisfied.

Let $u_{n}: \mathbb{N}^{*} \rightarrow \mathbb{N}^{*}$ and $Q_{n}: \mathbb{N}^{*} \rightarrow \mathbb{N}$ be as in Lemma 2.2. Now we define $\Gamma_{n} \subseteq \mathbb{N}^{*}$ by

$$
\begin{aligned}
& \Gamma_{0}=\mathbb{N}^{*}, \\
& \Gamma_{1}=\bigcup_{\omega \in \Gamma_{0}} u_{1}(\omega) \underbrace{Z_{1} \ldots Z_{1}}_{Q_{1}(\omega) \text { times }}, \\
& \Gamma_{2}=\bigcup_{\omega \in \Gamma_{1}} u_{2}(\omega) \underbrace{Z_{2} \ldots Z_{2}}_{Q_{2}(\omega) \text { times }}, \quad \ldots
\end{aligned}
$$

and

$$
E_{n}=\bigcup_{\omega \in \Gamma_{n}} \pi([\omega])
$$

Finally, let

$$
E=\bigcap_{n} E_{n}
$$

We will now prove that $E$ has the properties (1)-(3) listed before Lemma 2.1.

We first prove that $Z_{n}$ is non-empty for all $n$. In order to prove this we will need the following result from [O11]. For $x \in[0,1]$, let

$$
x=\sum_{n=1}^{\infty} \frac{\varepsilon_{N, n}(x)}{N^{n}},
$$

where $\varepsilon_{N, n}(x) \in\{0,1, \ldots, N-1\}$ for all $n$, denote the unique non-terminating $N$-adic expansion of $x$. For a positive integer $n$ and a finite string $\mathbf{i}=i_{1} \ldots i_{k} \in\{0,1, \ldots, N-1\}^{k}$ we write

$$
\Lambda_{N}(x, \mathbf{i} ; n)=\frac{\left|\left\{1 \leq i \leq n \mid \varepsilon_{N, i}(x)=i_{1}, \ldots, \varepsilon_{N, i+k-1}(x)=i_{k}\right\}\right|}{n}
$$

for the frequency of the string $\mathbf{i}$ among the first $n$ digits in the $N$-adic expansion of $x$, and let

$$
\Lambda_{N}^{k}(x ; n)=\left(\Lambda_{N}(x, \mathbf{i} ; n)\right)_{\mathbf{i} \in\{0,1, \ldots, N-1\}^{k}}
$$


denote the vector of frequencies $\Lambda_{N}(x, \mathbf{i} ; n)$ of all strings $\mathbf{i} \in\{0,1, \ldots, N-1\}^{k}$ of length $k$. Let

$$
\Delta_{N}^{k}=\left\{\left(p_{\mathbf{i}}\right)_{\mathbf{i} \in\{0,1, \ldots, N-1\}^{k}} \mid p_{\mathbf{i}} \geq 0, \sum_{\mathbf{i}} p_{\mathbf{i}}=1\right\}
$$

and

$$
\begin{aligned}
\mathbf{S}_{N}^{k}=\left\{\left(p_{\mathbf{i}}\right)_{\mathbf{i} \in\{0,1, \ldots, N-1\}^{k}} \mid p_{\mathbf{i}} \geq 0,\right. & \sum_{\mathbf{i}} p_{\mathbf{i}}=1, \sum_{i} p_{i \mathbf{i}}=\sum_{i} p_{\mathbf{i} i} \\
& \text { for all } \left.\mathbf{i} \in\{0,1, \ldots, N-1\}^{k-1}\right\},
\end{aligned}
$$

i.e. $\Delta_{N}^{k}\left(\mathrm{~S}_{N}^{k}\right)$ denotes the simplex of (shift invariant) probability vectors indexed by strings $\mathbf{i}=i_{1} \ldots i_{k}$ of length $k$ with entries $i_{j} \in\{0,1, \ldots, N-1\}$. Define $H_{N}^{k}: \Delta_{N}^{k} \rightarrow \mathbb{R}$ by

$$
H_{N}^{k}(\mathbf{p})=-\frac{1}{\log N} \sum_{\mathbf{i} \in\{0,1, \ldots, N-1\}^{k-1}} \sum_{i} p_{\mathbf{i} i} \log \frac{p_{\mathbf{i} i}}{\sum_{j} p_{\mathbf{i} j}}
$$

for $\mathbf{p}=\left(p_{\mathbf{i}}\right)_{\mathbf{i} \in\{0,1, \ldots, N-1\}^{k}}$ (as usual, we put $0 \log 0=0$ ). The following result is proved in [Ol1, Theorem 1].

Theorem 2.3. Let $\mathbf{p} \in \Delta_{N}^{k}$.

(1) If $\mathbf{p} \notin \mathrm{S}_{N}^{k}$, then

$$
\left\{x \in[0,1] \mid \lim _{n}\left\|\Lambda_{N}^{k}(x ; n)-\mathbf{p}\right\|_{1}=0\right\}=\emptyset .
$$

(2) If $\mathbf{p} \in \mathrm{S}_{N}^{k}$, then

$$
\operatorname{dim}\left\{x \in[0,1] \mid \lim _{n}\left\|\Lambda_{N}^{k}(x ; n)-\mathbf{p}\right\|_{1}=0\right\}=H_{N}^{k}(\mathbf{p}) .
$$

We can now prove that $Z_{n}$ is non-empty.

LEMMA 2.4. $Z_{n} \neq \emptyset$ for all $n$.

Proof. Recall that $\mathbf{q}=\left(q_{\mathbf{i}}\right)_{\mathbf{i} \in \mathbb{N}^{k}}$ where $\underset{q_{\mathbf{i}}}{q}=0$ for $\mathbf{i} \in \mathbb{N}^{k} \backslash\{1, \ldots, N\}^{k}$. For $\mathbf{i}=i_{1} \ldots i_{k} \in\{0,1, \ldots, N-1\}^{k}$, write $\widetilde{\mathbf{i}}=\widetilde{i}_{1} \ldots \widetilde{i}_{k} \in\{1, \ldots, N\}^{k}$ where $\widetilde{i}_{j}=i_{j}+1$. Define $\widetilde{\mathbf{q}}$ by

$$
\widetilde{\mathbf{q}}=\left(q_{\mathbf{i}}\right)_{\mathbf{i} \in\{0,1, \ldots, N-1\}^{k}} .
$$

Also, define $\widetilde{\mathrm{P}}(\omega)$ for $\omega \in \bigcup_{n} \mathbb{N}^{n+k-1}$ by

$$
\widetilde{\mathrm{P}}(\omega)=(\mathrm{P}(\omega, \widetilde{\mathbf{i}}))_{\mathbf{i} \in\{0,1, \ldots, N-1\}^{k}} .
$$

Finally, let

$$
X=\left\{\omega \in \bigcup_{l \geq k n N^{k}}\{1, \ldots, N\}^{l} \mid\|\widetilde{\mathrm{P}}(\omega)-\widetilde{\mathbf{q}}\|_{1} \leq \frac{1}{n}\right\} .
$$


It is clear that $X \subseteq Z_{n}$. We now claim that $X \neq \emptyset$. Since $\mathbf{q}=\left(q_{\mathbf{i}}\right)_{\mathbf{i} \in \mathbb{N}^{k}} \in \mathrm{S}_{k}$ and $q_{\mathbf{i}}=0$ for $\mathbf{i} \in \mathbb{N}^{k} \backslash\{1, \ldots, N\}^{k}$, we conclude that $\widetilde{\mathbf{q}} \in \mathrm{S}_{N}^{k}$, and it therefore follows immediately from Theorem 2.3 that

$$
\operatorname{dim}\left\{x \in[0,1] \mid \lim _{n}\left\|\Lambda_{N}^{k}(x ; n)-\widetilde{\mathbf{q}}\right\|_{1}=0\right\}=H_{N}^{k}(\widetilde{\mathbf{q}})>0,
$$

whence $\left\{x \in[0,1] \mid \lim _{n}\left\|\Lambda_{N}^{k}(x ; n)-\widetilde{\mathbf{q}}\right\|_{1}=0\right\} \neq \emptyset$. We can thus choose $x \in$ $[0,1]$ such that $\lim _{n}\left\|\Lambda_{N}^{k}(x ; n)-\widetilde{\mathbf{q}}\right\|_{1}=0$. Put $\omega=\omega_{1} \omega_{2} \ldots \in\{1, \ldots, N\}^{\mathbb{N}}$ where $\omega_{i}=\varepsilon_{N, i}(x)+1$. Then $\omega \mid m$ lies in $X$ for $m$ large enough.

Proposition 2.5. $E \subseteq \mathbb{E}_{k, m}$.

Proof. Let $x \in E$. We must now find a sequence $\left(n_{l}\right)_{l}$ of integers with $\lim _{l} n_{l}=\infty$ such that $\left\|\Pi_{k}\left(x ; n_{l}\right)-\mathbf{q}\right\|_{1} \rightarrow 0$. Since $x \in E=\bigcap_{n} E_{n}$, we conclude that for each positive integer $n$, we can find $\gamma_{n} \in \Gamma_{n}$ such that $x \in$ $\pi\left(\left[\gamma_{n}\right]\right)$. We now define the sequence $\left(n_{l}\right)_{l}$ by $n_{l}=\left|\gamma_{l}\right|-(k-1)$ and claim that $n_{l} \rightarrow \infty$ and $\left\|\Pi_{k}\left(x ; n_{l}\right)-\mathbf{q}\right\|_{1} \rightarrow 0$. We first prove that $n_{l} \rightarrow \infty$. However, this is obvious since $\gamma_{l} \in \Gamma_{l}$. Next, we prove that $\left\|\Pi_{k}\left(x ; n_{l}\right)-\mathbf{q}\right\|_{1} \rightarrow 0$. It follows from (2.4) and the definition of $\Gamma_{n}$ that $\Gamma_{n} \subseteq \widehat{Z}_{n}$ for all $n$. In particular, we conclude that $\gamma_{l} \in \Gamma_{l} \subseteq \widehat{Z}_{l}$. Using this and the fact that $\Pi_{k}\left(x ; n_{l}\right)=\mathrm{P}_{k}\left(\gamma_{l}\right)$, we conclude that

$$
\left\|\Pi_{k}\left(x ; n_{l}\right)-\mathbf{q}\right\|_{1}=\left\|\mathbf{P}_{k}\left(\gamma_{l}\right)-\mathbf{q}\right\|_{1} \leq 5 / l \rightarrow 0 .
$$

Proposition 2.6. $E$ is dense in $\mathbb{P}$.

Proof. Let $x \in \mathbb{P}$ and $r>0$. We must now find $t \in E \cap B(x, r)$. We first observe that there exists $\omega \in \mathbb{N}^{*}$ such that

$$
\pi([\omega]) \subseteq B(x, r) .
$$

Next, since $Z_{n} \neq \emptyset$ for all $n$ (cf. Lemma 2.4), we can choose strings $\omega_{n} \in \mathbb{N}^{*}$ inductively as follows. Let

$$
\begin{aligned}
& \omega_{0}=\omega \in \Gamma_{0}, \\
& \omega_{1} \in u_{1}\left(\omega_{0}\right) \underbrace{Z_{1} \ldots Z_{1}}_{Q_{1}\left(\omega_{0}\right) \text { times }} \subseteq \Gamma_{1}, \\
& \omega_{2} \in u_{2}\left(\omega_{1}\right) \underbrace{Z_{2} \ldots Z_{2}}_{Q_{2}\left(\omega_{1}\right) \text { times }} \subseteq \Gamma_{2},
\end{aligned}
$$

For each $n$ we have

$$
\pi\left(\left[\omega_{n+1}\right]\right)^{-} \subseteq \pi\left(\left[u_{n}\left(\omega_{n}\right)\right]\right)^{-} \subseteq \pi\left(\left[\omega_{n}\right]\right)^{\circ} \subseteq \pi\left(\left[\omega_{n}\right]\right) \subseteq \pi\left(\left[\omega_{n}\right]\right)^{-} .
$$

In (2.7), the closure and interior are with respect to the space $\mathbb{P}$. It follows from (2.7) that

$$
\bigcap_{n} \pi\left(\left[\omega_{n}\right]\right)^{-}=\bigcap_{n} \pi\left(\left[\omega_{n}\right]\right)
$$


It also follows from $(2.7)$ that $\left(\pi\left(\left[\omega_{n}\right]\right)^{-}\right)_{n}$ is a decreasing sequence of nonempty compact subsets of $[0,1]$, and the intersection $\bigcap_{n} \pi\left(\left[\omega_{n}\right]\right)^{-}$is therefore non-empty. Now pick any $t \in \bigcap_{n} \pi\left(\left[\omega_{n}\right]\right)^{-}$. We claim that $t \in E \cap B(x, r)$. We first prove that $t \in E$. Using (2.8) we see that $t \in \bigcap_{n} \pi\left(\left[\omega_{n}\right]\right)^{-}=$ $\bigcap_{n} \pi\left(\left[\omega_{n}\right]\right) \subseteq \bigcap_{n} E_{n}=E$. Next we prove that $t \in B(x, r)$. We clearly have (using (2.6)) $t \in \pi\left(\left[\omega_{0}\right]\right)=\pi([\omega]) \subseteq B(x, r)$.

Proposition 2.7. $E$ is a $\mathcal{G}_{\delta}$ set.

Proof. For a positive integer $n$ we define the set $G_{n}$ by

$$
G_{n}=\bigcup_{\omega \in \Gamma_{n}} \pi([\omega])^{\circ}
$$

where there interior is with respect to the space $\mathbb{P}$. The set $G_{n}$ is clearly open (in $\mathbb{P}$ ). We now have

$$
\begin{aligned}
E_{n+1} & =\bigcup_{\omega \in \Gamma_{n+1}} \pi([\omega]) \subseteq \bigcup_{\sigma \in \Gamma_{n}} \pi\left([u_{n+1}(\sigma) \underbrace{Z_{n+1} \ldots Z_{n+1}}_{Q_{n+1}(\sigma) \text { times }}]\right) \\
& \subseteq \bigcup_{\sigma \in \Gamma_{n}} \pi\left(\left[u_{n+1}(\sigma)\right]\right) \subseteq \bigcup_{\sigma \in \Gamma_{n}} \pi([\sigma])^{\circ}=G_{n},
\end{aligned}
$$

and

$$
G_{n}=\bigcup_{\omega \in \Gamma_{n}} \pi([\omega])^{\circ} \subseteq \bigcup_{\omega \in \Gamma_{n}} \pi([\omega])=E_{n}
$$

It follows immediately from (2.9) and (2.10) that $E=\bigcap_{n} E_{n}=\bigcap_{n} G_{n}$. Since each $G_{n}$ is open, this shows that $E$ is $\mathcal{G}_{\delta}$.

Proof of Theorem 1. (1) Since $\mathbb{P}$ is a Baire space, it follows immediately from Propositions 2.5-2.7 that the set $\mathbb{E}$ is comeager.

(2) This statement easily follows from Theorem 1(1).

\section{References}

[Bi] P. Billingsley, Ergodic Theory and Information, Wiley, 1965.

[Ed] G. Edgar, Integral, Probability, and Fractal Measures, Springer, New York, 1998.

[Fa] K. J. Falconer, Fractal Geometry, Wiley, 1990.

[KN] L. Kuipers and H. Niederreiter, Uniform Distribution of Sequences, Wiley-Interscience, New York, 1974.

[Lé] P. Lévy, Théorie de l'addition des variables aléatoires, Gauthier-Villars, Paris, 1937.

[Ol1] L. Olsen, Applications of multifractal divergence points to sets of numbers defined by their $N$-adic expansion, Math. Proc. Cambridge Philos. Soc., to appear.

[Ol2] - Extremely non-normal numbers, preprint, 2002. 
[Ša1] T. Šalát, Zur metrischen Theorie der Lürothschen Entwicklungen der reellen Zahlen, Czechoslovak Math. J. 93 (1968), 489-522.

[Ša2] —, Bemerkung zu einem Satz von P. Lévy in der metrischen Theorie der Kettenbrüche, Math. Nachr. 41 (1969), 91-94.

Department of Mathematics

University of St. Andrews

St. Andrews, Fife KY16 9SS, Scotland

E-mail: lo@st-and.ac.uk 